REGARDS

SUR LECONOMIE ALLEMANDE

BULLETIN ECONOMIQUE DU CRAC

\section{Regards sur l'économie allemande}

Bulletin économique du CIRAC

$68 \mid 2004$

Varia

\title{
Hartz IV : la fin des trappes à inactivité ?
}

Isabelle Bourgeois

\section{(2) OpenEdition}

\section{Journals}

Édition électronique

URL : http://journals.openedition.org/rea/3618

DOI : 10.4000/rea.3618

ISBN : 978-2-8218-0832-4

ISSN : 1965-0787

Éditeur

CIRAC

Édition imprimée

Date de publication : 1 octobre 2004

Pagination : 5-12

ISSN : 1156-8992

Référence électronique

Isabelle Bourgeois, « Hartz IV : la fin des trappes à inactivité ? », Regards sur l'économie allemande [En ligne], 68 | octobre 2004, mis en ligne le 29 avril 2009, consulté le 01 mai 2019. URL : http:// journals.openedition.org/rea/3618; DOI : 10.4000/rea.3618 


\section{Hartz IV : \\ la fin des trappes à inactivité ?}

\section{Isabelle Bourgeois}

Le dernier volet des réformes du marché de l'emploi : la loi Hartz IV, qui entrera en vigueur le 01-01-2005, a suscité cet été un cortège de manifestations dans l'est de l'Allemagne, orchestrées par les extrêmes politiques protestant contre le " démantèlement " de l'Etat-prévoyance. II n'en est rien pourtant, bien au contraire. Pour combattre le chômage de longue durée, la réforme supprime l'un des trois étages du régime d'indemnisation : I'Arbeitslosenhilfe, une allocation versée aux chômeurs à l'expiration de leurs droits à l'assurance. Financée par l'impôt, elle relevait du principe d'assistance et relayait les indemnités chômage stricto sensu (Arbeitslosengeld) qui, financées par les cotisations, relèvent, elles, de la logique d'assurance. Ne subsisteront donc en 2005 que deux étages : l'Arbeitslosengeld, désormais relayé au bout d'un an par un mécanisme d'aide surnommé Arbeitslosengeld II. La réforme modifie profondément la logique du système d'indemnisation : tout bénéficiaire d'un des deux revenus de substitution est désormais considéré comme une personne en quête active et effective d'emploi. Ce changement corrige les dérives de l'Etat-providence allemand dont la générosité avait abouti en quarante ans à générer ces trappes à inactivité (voir REA 51/01) qui incitent aujourd'hui quelque trois millions de personnes à s'installer dans le chômage.

La "Quatrième loi pour des services modernes du marché de l'emploi », du 2412-2003, surnommée Hartz IV, modifie pour l'essentiel le chapitre II du Code social (Sozialgesetzbuch), substituant à l'ancienne Arbeitslosenhilfe une nouvelle prestation : le " minimum garanti pour les personnes en quête d'emploi » (Grundsicherung für Arbeitsuchende). Elle vise à «renforcer la responsabilité individuelle des personnes nécessiteuses à capacité de travail entière, comme de ceux qui vivent avec elles dans une communauté de besoins, et à contribuer à ce qu'elles soient capables d'assurer leur subsistance indépendamment du minimum garanti, c'est-à-dire par leurs propres forces et moyens » $(\S 1,1)$. Ce minimum garanti se compose de deux types de prestations, destinées pour le premier à "réduire l'état de nécessité ou à lui mettre fin notamment par l'insertion en emploi » et, pour le second, à « assurer la subsistance » (\$2).

Un retour aux sources de l'économie sociale de marché.

La Grundsicherung für Arbeitsuchende repose sur un principe fondateur de l'économie sociale de marché : l'aide à l'auto-assistance (Hilfe zur Selbsthilfe). Pour corriger les effets négatifs du marché, la collectivité a un devoir de solidarité envers l'individu, l'individu un droit à cette solidarité - mais seulement s'il ne parvient pas à surmonter seul ses difficultés. C'est là l'expression la plus pure du principe de subsidiarité qui interdit à l'Etat de se substituer à la responsabilité individuelle. Ce principe caractérisait déjà l'aide sociale (Sozialhilfe) adoptée en 1962, mais avait fini par se diluer avec la progression du chômage. Dès 1982, Otto Graf Lambsdorff (FDP), ministre des finances du chancelier Helmut Schmidt (SPD), évoquait les risques pour la croissance et l'emploi d'un "engrenage de répartition qui, en pénalisant toujours plus la performance et la prévoyance individuelles, entretient le développement d'une logique de revendication et d'une mentalité d'assisté ». C'est avec cet engrenage que cherche à rompre la loi Hartz IV.

L'Arbeitslosengeld I/ est donc littéralement un revenu minimum d'insertion avec ses deux volets : mesures de (re)qualification et d'insertion, soutien pécuniaire. La différence avec le régime précédent est principalement de deux ordres.

Une sorte de RMI fonction du degré de nécessité... 
... avec obligation d'accepter emploi ou formation proposés

... et déconnectée du nouveau régime de l'aide sociale
D'abord, le revenu de substitution de base est un forfait (en règle générale: $345 €$ par mois à l'ouest, $331 €$ à l'est) à l'inverse de l'Arbeitslosenhilfe qui, sur le modèle des allocations chômage (Arbeitslosengeld), était fonction du salaire net antérieur (53\% pour un célibataire sans enfant, $57 \%$ avec enfant). Ensuite, le montant auquel peut prétendre le bénéficiaire est fonction du degré individuel de nécessité (Bedürftigkeit) : entrent donc en ligne de compte dans le calcul des prestations toutes les sources de revenus (revenus immmobiliers, par exemple), ainsi que le patrimoine (immobilier, épargne, etc.) du chômeur comme de ses proches - mais au-delà d'un certain plafond seulement. Ce critère de nécessité était spécifique à l'aide sociale (Sozialhilfe) versée par les communes aux personnes en difficulté au titre de la lutte préventive contre l'exclusion. Le nouveau minimum garanti s'inspire donc de l'aide sociale en déconnectant la prestation financière du salaire précédent, son versement intégral étant en outre fonction de la situation patrimoniale du bénéficiaire. Dans le même temps, il lie l'ouverture des droits à l'obligation de rechercher activement un emploi. C'est une des raisons pour lesquelles, pour décrire la loi Hartz IV, on évoque une fusion entre aide au chômeur en fin de droits et aide sociale.

Sont considérés comme bénéficiant d'une capacité de travail pleine et entière toutes les personnes de 15 à 65 ans susceptibles de travailler au moins trois heures par jour; l'âge de 15 ans correspond à l'entrée dans la vie active via l'apprentissage, 65 ans à l'âge légal de départ à la retraite. L'obligation qui leur est faite de chercher activement un emploi est contractualisée. A cet effet, elles signent avec leur conseiller de l'Agence locale pour l'emploi une convention d'insertion (Eingliederungsvereinbarung) de six mois, renouvelable autant de fois que l'exige l'état de nécessité. Mais le chômeur est censé retrouver assez vite du travail car « tout emploi est acceptable » (zumutbar) et doit être accepté dès lors que le bénéficiaire est psychiquement et physiquement en mesure de l'occuper (§10). Cela vaut même si l'emploi implique un changement de métier ou ne correspond pas à la qualification initiale et si la rémunération est inférieure au salaire conventionnel en vigueur dans la branche ou dans la région. Seules exceptions : la charge de parents dépendants ou d'un enfant de moins de trois ans lorsqu'il n'est pas inscrit dans une crèche ou un jardin d'enfant (mais l'Agence doit alors aider le candidat à trouver une structure d'accueil afin de le rendre 'employable'). En cas de refus de l'emploi, de la mesure d'insertion proposée ou de non présentation, le bénéficiaire est informé des conséquences de son " comportement improductif » $(\$ 31,3-2)$ et encourt, en cas de récidive, des sanctions allant de la réduction du montant du minimum garanti (30\%) à la radiation ; ces sanctions sont toutefois limitées à une durée de 3 mois.

Les personnes à capacité de travail réduites ou démunies bénéficieront, elles, de l'aide sociale. L'ancienne Sozialhilfe a en effet été maintenue dans son principe, mais son champ d'application a été réduit aux seules personnes se trouvant dans l'incapacité d'assurer leur subsistance par leur propres forces et moyens. A cet effet, la loi fédérale Bundessozialhilfegesetz de 1962 a été modifiée en plusieurs étapes, indépendamment de la loi Hartz IV, le nouveau texte étant intégré dans le Code social allemand dont il constitue désormais le chapitre XII, intitulé Sozialhilfe. Le principe de cette aide à l'auto-assistance est maintenu : "permettre aux bénéficiaires de mener une vie conforme à la dignité humaine » (SGB XII, §1). L'aide, versée par les communes et prélevée sur leurs recettes fiscales, vise la prévention de l'exclusion sociale. Ultime 'filet social', elle n'intervient qu'en dernier recours, c'est-à-dire après épuisement de tous les moyens permettant normalement à un individu d'assurer sa subsistance : travail, patrimoine, revenus des proches, droits ouverts au titre de la protection sociale - elle est donc elle aussi soumise au critère de nécessité. Mais elle est automatique : la personne dans le besoin n'a pas à en faire la demande, les services sociaux des communes s'autosaisissent de son dossier. Les cas sont en effet connus, les personnes soutenues ayant généralement une longue antériorité de prestations. 
L'aide matérielle qui leur est versée est un « minimum socio-culturel vital », d'un montant forfaitaire de $345 €$ à l'ouest, de $331 €$ à l'est à partir du $1^{\text {er }}$ janvier 2005 pour un célibataire ou le chef de famille, à quoi s'ajoutent le cas échéant une aide forfaitaire aux enfants présents dans le ménage. Ce minimum, établi en fonction de besoins moyens, sera recalculé tous les cinq ans sur la base des dépenses des ménages dans les tranches inférieures de revenus recensées par Destatis (Enquête EVS, voir REA 67/04). Le cas échéant, six types d'aides spécifiques peuvent s'y ajouter, mais il revient alors au bénéficiaire du minimum vital d'en faire la demande: un minimum vieillesse, un complément maladie (tous les bénéficiaires de la Sozialhilfe sont affiliés au système d'assurance maladie public depuis le 01-01-2004; et ils auront une assurance retraite dès le 01-01-2005), une aide à l'insertion des personnes handicapées, une aide aux personnes dépendantes (en complément à l'assurance dépendance, voir dans ce numéro), une aide aux personnes en difficulté particulière (SDF, par exemple), et une aide en cas de situation exceptionnelle (obsèques, etc.).

\section{2,81 millions de bénéficiaires de l'aide sociale en 2003, dont 836000 chômeurs}

A la fin 2003, Destatis avait recensé 2,81 millions de personnes percevant le « minimum socioculturel vital » de l'aide sociale ancienne manière, soit $3,4 \%$ de la population.

Dans la population étrangère, le taux $(8,4 \%)$ est supérieur à ce qu'il est chez les Allemands $(2,9 \%)$. Mais l'évolution depuis 1991 de la structure des bénéficiaires révèle que la part des étrangers reste stable sur le long terme (elle oscille entre 567 000, le minimum de 1991 et 758000 , le maximum, enregistré en 1992 ; en 2003, elle est de 617000 ), à l'inverse de celle des Allemands, passée continûment de 1,469 million à 2,194 millions en 13 ans.

Chez les femmes, le taux est de 3,7\% (contre 3,1\% chez les hommes): un tiers des femmes élevant seule leur enfant bénéficie de la Sozialhilfe, la collectivité se substituant au conjoint absent au titre du soutien familial (voir REA 51/01). La part des bénéficiaires de moins de 18 ans en est le reflet : ils sont 1,08 million. Quant à la part des plus de 65 ans, elle a diminué de $-48 \%$ en un an (-98 000 personnes) : depuis l'entrée en vigueur au 01-01-2003 de la loi Gesetz über eine bedarfsorientierte Grundsicherung im Alter und bei Erwerbsminderung (GSiG) qui instaure une sorte de minimum vieillesse (ou handicap) garanti destiné à assurer la subsistance des personnes dépendantes non couvertes par l'assurance dépendance, cette prestation se substitue normalement à l'aide sociale. $0,7 \%$ des plus de 65 ans continuent cependant de percevoir parallèlement l'aide sociale pour couvrir des besoins particuliers (alimentation spécifique, par exemple) ou parce que leur conjoint est bénéficiaire de l'aide sociale. Le nouveau régime de la Sozialhilfe ne devrait guère modifier cette situation.

Dans la tranche d'âge des 18-64 ans, Destatis dénombre 1,63 million de bénéficiaires. En un an, ce groupe a augmenté de $+5,3 \%$ du fait de l'arrivée massive de personnes parallèlement inscrites au chômage : 836000 très exactement, soit près de la moitié (47\%) des bénéficiaires en âge de travailler (15-64 ans). Un gros tiers (36,1 \%) d'entre elles percevaient à la fois des indemnités chômage (les deux types existant jusqu'au 31-12-2004) et l'aide sociale. Elles ne pourront plus prétendre à l'aide sociale à partir du $1^{\text {er }}$ janvier 2005.

L'écrasante majorité des bénéficiaires de l'aide sociale est domiciliée dans les Länder ouestallemands (hors Berlin), 426000 seulement dans les nouveaux Länder (hors Berlin). Le plus fort taux d'aide sociale est enregistré dans les Länder de Brême $(9,2 \%)$, Berlin $(7,7 \%)$ et Hambourg $(6,9 \%)$. Les taux les plus bas sont enregistrés en Bavière $(1,8 \%)$ et dans le Bade-Wurtemberg $(2,1 \%)$, ainsi que dans la dynamique Thuringe $(2,3 \%)$.

Depuis 1962, le périmètre de la Sozialhilfe était devenu protéiforme. Destinée à l'origine à aider momentanément quelques rares personnes en difficulté, surtout les retraités à faibles revenus, l'aide s'était transformée au fil des mutations économiques et sociales en un mécanisme de soutien massif, indifférencié et souvent pérenne à de nouvelles populations : mères célibataires, chômeurs de longue durée, chômeurs de plus de 55 ans, actifs étrangers faiblement qualifiés, jeunes chômeurs sans couverture maladie, demandeurs d'asile, rapatriés est-européens de souche allemande, personnes âgées dépendantes, handicapés... Une série de lois avait tenté de remédier à cette situation qui pesait de plus en plus sur les budgets communaux : en 1963, pour 500000 bénéficiaires, l'aide sociale ne représentait que $2,7 \%$ des dépenses des communes ; depuis 1997, pour près de 3 millions de bénéficiaires, elle en accapare 6 à $7 \%$. Des régimes spécifiques avaient alors été créés pour certains types de population, dont les demandeurs d'asile (1993) ou les personnes dépendantes (création de l'assurance dépendance en 1995).
Sozialhilfe : un « minimum socio-culturel vital »

Une Sozialhilfe resserrée pour mettre fin aux dérives 
La fin du cumul des aides

Une stricte séparation entre Sozialhilfe et Arbeitslosengeld II

Une nouvelle définition du chômeur

3,4 millions de personnes sans emploi depuis 12 mois
Restait le cœur du problème, auquel s'attaquent aujourd'hui conjointement le nouveau chapitre XII du Code social et la loi Hartz IV : le cumul de l'aide sociale et de l'aide aux chômeurs en fin de droits, qui garantissait au bénéficiaire un revenu de substitution suffisant pour qu'il ne soit guère intéressant de reprendre un emploi. En instaurant de facto un revenu minimum implicite, il contribuait à générer l'une des principales trappes à inactivité à l'origine du socle structurel de chômage de longue durée (50 \% des sans emploi sont au chômage durant plus de 12 mois). Cette situation était en outre entretenue par la coexistence de deux types d'acteurs indépendants: les communes finançant et gérant l'aide sociale via leurs bureaux des affaires sociales, les Agences locales pour l'emploi dispensant l'aide aux chômeurs en fin de droits, financée par le Bund, mais administrée en pleine autonomie par l'Office de Nuremberg. Cela prolongeait d'autant la durée de l'assistance, les deux acteurs se renvoyant les candidats à intervalles réguliers. Or la moitié des bénéficiaires de l'aide sociale est considérée comme capable de (re)prendre une activité, estimait Destatis dans un rapport publié en février 2001, ajoutant: " une réintégration de ces personnes dans le marché de l'emploi aurait vraisemblablement pour effet que nombre des membres de leur famille n'auraient plus besoin de la Sozialhilfe ».

Cette réintégration est l'objet exprès de la loi Hartz IV. Certes, le montant forfaitaire automatique de la Sozialhilfe est le même que celui du nouvel Arbeitslosengeld II ; c'est l'autre raison pour laquelle on évoque souvent, à propos de la loi Hartz IV, une fusion entre aide sociale et aide au chômeur en fin de droits. Mais en réalité, ce terme est impropre, car l'articulation entre les deux régimes les différencie clairement: l'Arbeitslosengeld /l s'adresse aux personnes en quête active d'emploi, la Sozialhilfe aux personnes en mal d'insertion sociale. La frontière entre les deux groupes est la capacité de travail : supérieure à 3 heures par jour pour le premier, inférieure pour le second. La loi Hartz IV sort donc du périmètre de l'aide sociale tous les chômeurs qui pouvaient y prétendre précédemment. Ils bénéficieront du nouvel Arbeitslosengeld II.

D'autre part, le nouveau régime instauré par la loi renvoie à une nouvelle définition du 'statut' du chômeur, qui équivaut à une mise en conformité de l'approche allemande avec la pratique de l'OIT en vigueur en Europe. La différence essentielle porte sur deux critères : la disponibilité (obligation de se présenter tous les trois mois à l'Agence selon l'ancienne loi allemande ; disponibilité sous 15 jours pour l'OIT) et la notion de quête d'emploi (quête active selon l'OIT alors que la seule inscription à l'ANPE allemande équivalait à une demande d'emploi ; voir REA 59/02). En chiffres, elle se soldait par près de 800000 personnes en plus (données de l'Agence de Nuremberg) ou en moins (données Destatis et Eurostat établies selon les critères OIT), soit une différence de près d'un point de pourcentage entre les taux de chômage publiés par les diverses instances. Pour ne pas s'attirer le reproche de « maquiller » les statistiques du chômage, le gouvernement fédéral a peu communiqué sur cette européanisation du régime allemand de protection contre le chômage. En outre, il s'agissait d'un dossier complexe impliquant une réforme du Code social, à l'origine de la généreuse définition allemande, et une restructuration de l'Office fédéral du travail, aujourd'hui rebaptisé Agence fédérale pour l'emploi (Bundesagentur für Arbeit, $B A$ ) afin de concrétiser aussi le changement d'approche ; depuis le 10-092004, la BA met en œuvre les méthodes d'enquête téléphonique de l'OIT. Dans le vaste chantier de réforme du marché de l'emploi lancé par l'Agenda 2010, la loi Hartz IV se contente ainsi d'appliquer aux chômeurs de longue durée les critères européens de disponibilité et de quête active d'un emploi.

Le nombre de chômeurs concernés dès le $1^{\text {er }}$ janvier 2005 par le nouveau minimum garanti de l'Arbeitslosengeld // est estimé par le gouvernement fédéral à 3,2 millions. L'institut de recherche IAB de l'Agence de Nuremberg table, pour sa part, sur 3,4 millions de bénéficiaires potentiels. Ce nombre est la somme des chômeurs parallèlement bénéficiaires de la Sozialhilfe ancienne manière, des anciens bénéficiaires de l'aide sociale capables de travailler au moins 3 
heures par jour, des chômeurs indemnisés jusqu'ici par le régime de l'assurance chômage mais qui arrivent en fin de droits, ainsi que des bénéficiaires de l'ancienne Arbeitslosenhilfe, abolie par la loi Hartz IV. La liste des personnes concernées reflète l'empilement des diverses mesures d'aide et d'indemnisation auquel met fin la réforme pour réduire la durée du chômage - objectif prioritaire. Tous sont sans emploi depuis au moins 12 mois.

\section{Les réformes du marché du travail de l'Agenda 2010 : les lois Hartz I à IV}

A l'issue des travaux de la Commission Services modernes sur le marché de l'emploi (Moderne Dienstleistungen am Arbeitsmarkt) instituée au début de 2002 et présidée par Peter Hartz, le DRH de VW (voir REA 59/02) - d'où son surnom de Commission Hartz -, quatre lois de réformes ont été adoptées par le parlement allemand, dans le contexte de l'Agenda 2010, pour moderniser la politique de l'emploi et améliorer le placement des chômeurs : les « lois pour des services modernes sur le marché de l'emploi » (Gesetze für moderne Dienstleistungen am Arbeitsmarkt), surnommées les lois Hartz.

- La loi Hartz I, entrée en vigueur pour l'essentiel de ses dispositions le 01-01-2003, crée des Personal Service Agenturen (PSA, Agences de services de personnel) sortes d'agences d'intérim subventionnées et chargées de l'insertion de chômeurs difficiles à placer (voir REA 62 et 65/04). Parallèlement, la loi assouplit la réglementation anti-licenciement (voir $R E A$ 62/04) et durcit le régime d'indemnisation des chômeurs, exigeant de leur part une plus grande mobilité ou l'acceptation d'un emploi à rémunération inférieure.

- La loi Hartz II, entrée en vigueur entre le 01-01 et le 01-04-2003, modifie les dispositions relatives aux petits boulots : recours à la subvention salariale (voir $R E A 60 / 03$ ), création des Ich-AG et des mini-jobs (voir REA 67/04).

- La loi Hartz III, entrée en vigueur le 01-01-04, vise pour l'essentiel la restructuration de l'Office fédéral du Travail de Nuremberg, rebaptisé Agence fédérale du Travail (Bundesagentur für Arbeit, $B A$ ) et la rationalisation du régime des prestations : par exemple, un chômeur bénéficie des allocations chômage à l'issue de 12 mois d'emploi sur les deux dernières années (au lieu de trois) ; il perd ses droits aux prestations si le cumul des périodes de carence atteint 21 semaines.

- La loi Hartz IV, adoptée le 24-12-2003 à l'issue d'une procédure de conciliation entre Bundesrat et Bundestag, et qui entrera en vigueur au 01-01-05, modifie les deux régimes d'assistance financés par l'impôt qu'étaient l'aide au chômeur (Arbeitslosenhilfe) et l'aide sociale (Sozialhilfe) : la première est supprimée, le nouveau revenu de substitution, forfaitaire, est ramené au niveau de l'aide sociale et devient un "minimum garanti pour les personnes en quête d'emploi » (Grundsicherung für Arbeitsuchende), communément appelé Allocations chômage II (Arbeitslosengeld II). II s'agira d'une sorte de revenu minimum d'insertion dont le versement est soumis au critère de la nécessité (Bedürftigkeit) et qui implique de la part du bénéficiaire une recherche active. La gestion en est confiée à la BA qui a dû augmenter son taux d'encadrement : passant d'un conseiller (Fallmanager) pour plus de 400 demandeurs d'emploi à un pour 75. Etant donné que les deux régimes d'assistance précédents étaient gérés par deux instances : la BA pour le premier, les communes pour le second, la loi crée des Jobs Centers, sortes de guichets uniques.

A terme, le changement le plus important affectera ceux qui percevaient les indemnités de l'assurance chômage (Arbeitslosengeld) : la loi Hartz IV limite en effet la durée du versement à 12 mois ; jusqu'ici, elle pouvait aller jusqu'à 32 mois, avant que l'ancienne aide au chômeur en fin de droits ne prenne le relais. Cette limitation ne concerne toutefois que les moins de 45 ans ; elle est allongée à 18 mois pour les plus de 55 ans, particulièrement affectés par le chômage de longue durée (63,6 \% des chômeurs de 50 à 64 ans ; Destatis). Mais cette mesure n'entrera en vigueur que le 01-02-2006. Pendant la période transitoire allant du 01-01-2005 au 31-01-2006, la durée de versement est échelonnée de 18 à 32 mois pour les chômeurs âgés de 47 à plus de 57 ans - dès lors qu'ils entrent au chômage pendant ce temps. En ce qui concerne le montant des indemnités, aucun changement : il s'élèvera toujours à $60 \%$ du dernier salaire net cumulé pour un célibataire, à $67 \%$ pour un chômeur avec enfant. Les moins de 47 ans en revanche qui entament leur $13^{\mathrm{e}}$ mois de chômage (ou plus) au début 2005 seront soumis au nouveau régime de l'Arbeitslosengeld II.

Mais les chômeurs arrivant en fin de droits ne seront pas livrés à une paupérisation brutale, contrairement à ce que laissent entendre les manifestants, qui se focalisent sur les $345 €$ ou $331 €$ mensuels. Car ce minimum garanti n'est qu'une prestation de base qui peut être augmentée, le cas échéant, d'une " allocation sociale » (Sozialgeld) pour enfant à charge (60\% du minimum de base pour les moins de 15 ans, puis $80 \%$ jusqu'à la majorité) ou pour le con-
Indemnités chômage versées pendant 12 mois au lieu de 32

Une transition en douceur entre ancien et nouveau régime 
1,5 million de bénéficiaires ne seront plus en état de «nécessité »

Epargne et patrimoine des revenus faibles et moyens sont protégés

$41 \%$ des nouvelles aides iront aux Länder de l'est joint en situation de nécessité (90\%), et d'une aide au logement (loyer et chauffage). Un célibataire dispose donc de $651 €$, un couple avec 2 enfants, de $1554 €$. A cela s'ajoute une «indemnité de transition » (Übergangsgeld). En 2005, elle s'élève aux deux tiers de la différence entre les indemnités perçues au préalable (plus, le cas échéant, l'aide au logement) et le nouveau minimum garanti (avec, le cas échéant, ses compléments). Ce montant est plafonné à $160 €$ par mois pour un célibataire, au double pour un couple, et à $60 €$ par enfant mineur à charge. Un célibataire perçoit donc $741 €$ (ouest ; salaire brut antérieur : $2000 €$ ). En 2006, ces montants seront réduits de moitié (696€ dans ce cas de figure). Au total, durant la phase de transition, les aides versées au titre du nouvel Arbeitslosengeld // sont très proches des prestations de l'ancienne Arbeitslosenhilfe (709 € pour le même célibataire) dont les anciens bénéficiaires ne sont donc pas pénalisés non plus. La différence ne sera sensible qu'en 2007 - mais le chômeur est censé avoir repris un emploi d'ici là.

C'est parmi les actuels bénéficiaires de l'Arbeitslosenhilfe (2,05 millions de personnes) que la situation sera la plus contrastée au $1^{\mathrm{er}}$ janvier $2005.9 \%$ d'entre eux ne verront aucun changement, a calculé la confédération syndicale DGB. $16 \%$ verront même s'améliorer leur situation: les anciens bénéficiaires de l'aide sociale qui auront désormais 'statut' de personne en quête d'emploi et qui bénéficieront d'une couverture sociale, de même que les familles nombreuses et ceux qui ont constitué un capital retraite (patrimoine 'intouchable'). 48 \% (un million de personnes) subiront une détérioration : ceux qui disposent d'un patrimoine ou de revenus leur permettant d'assurer partiellement leur subsistance. Enfin, $27 \%$ ne pourront plus prétendre à un quelconque soutien, puisqu'ils disposent de revenus (le salaire du conjoint, par exemple) ou d'un patrimoine suffisants. C'est là l'effet délibéré du calcul du degré de nécessité qui détermine l'ouverture des droits et le montant des aides versées.

L'Etat social allemand reste généreux. Les franchises et abattements, établis un peu sur le modèle de la fiscalité, sont importants ; les chômeurs ont droit à un patrimoine minimum - et il est conséquent. La loi protège particulièrement les ménages à revenus faibles et moyens, préservant notamment leur patrimoine immobilier. En effet, seuls $9 \%$ des foyers à l'ouest et $3 \%$ à l'est possèdent un logement dont la valeur vénale dépasse $200000 €$ (Destatis).

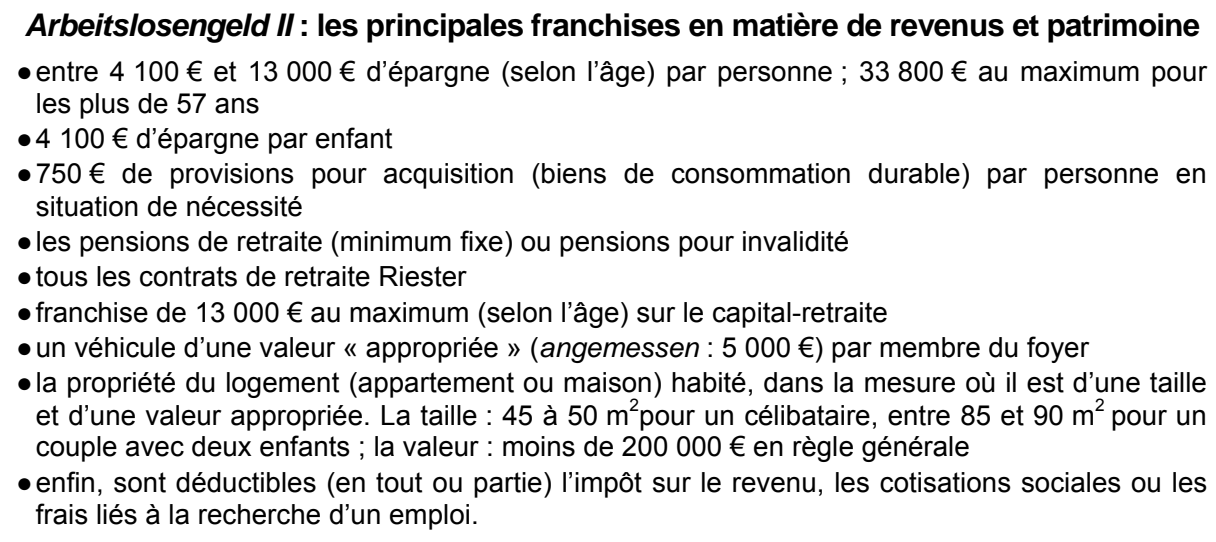

Le régime introduit par la loi Hartz IV tient compte également de la disparité des situations entre l'est et l'ouest du pays. En effet, proportionnellement, c'est dans les nouveaux Länder que les chômeurs de longue durée sont les plus nombreux : ils sont un million, soit la moitié du total des bénéficiaires de l'ancienne Arbeitslosenhilfe. Dans le cadre du soutien à l'effort de reconstruction de l'économie des nouveaux Länder, le Bund verse déjà annuellement 800 millions $€$ d'aides complémentaires spécifiques. S'y ajouteront désormais les moyens dédiés à la nouvelle politique de l'emploi (au total 6,35 milliards $€$ en 2005), dont $41 \%$ sont destinés aux régions où le taux global de chômage dépasse les 
$15 \%$. Celles-ci sont situées majoritairement à l'est de la RFA, mais aussi à l'ouest; ainsi, dans les régions en restructuration industrielle comme la Ruhr où, dans certaines villes (comme Duisburg), le taux de chômage est équivalent aux $20 \%$ enregistrés dans certaines agglomérations est-allemandes connaissant des problèmes similaires (Eisenhüttenstadt, par exemple). Ces moyens seront affectés à des emplois subventionnés (voir REA 60/03).

Le volet (ré)insertion du 'nouveau RMI' allemand ouvre aux bénéficiaires la possibilité d'exercer un emploi rémunéré parallèlement au versement des prestations. Le plafond des revenus (salaire et aides cumulés) à partir duquel les aides sont supprimées a été relevé à $1501 €$ bruts par mois ; pour un salaire (d'appoint) brut de $400 €$, les aides ne sont réduites que de $15 \%$. S'y ajoute la création de "jobs à un euro " (Ein-Euro-Jobs) : des travaux d'utilité collective proposés dans le secteur public ou caritatif et ouvrant droit à un dédommagement (non un salaire !) de 1 à $2 €$ par heure. Le bénéficiaire pourra ainsi 'arrondir ses fins de mois' jusqu'à concurrence de $330 €$. Le gouvernement fédéral espère que cette mesure permettra à 600000 personnes de garder ou de renouer le contact avec la vie sociale et professionnelle. Paradoxalement, ce ne sont pas les syndicats qui protestent, mais les fédérations de l'artisanat qui redoutent là l'émergence d'une concurrence « déloyale ».

Mais cette mesure comporte le risque de créer de nouvelles trappes à inactivité. L'Institut der deutschen Wirtschaft, proche de la fédération de l'industrie (BDI), a en effet calculé que, par l'effet cumulé des diverses prestations de l'Arbeitslosengeld I/ et de ces jobs à un euro, le revenu mensuel perçu était équivalent à celui d'un emploi rémunéré de 8 à $12 €$ l'heure. Dans le premier cas en effet, la somme des transferts (ouest) s'élève à $648 €$ pour un célibataire, à $1076 €$ pour un parent isolé (un enfant) et à $1554 €$ pour un couple avec deux enfants. Si on y ajoute $330 €$ issus d'un travail d'utilité collective, le total (non imposable) équivaut à un salaire mensuel brut (donc avant impôt) de $1350 €$ pour le célibataire et de $2050 €$ pour la famille. C'est plus que le salaire d'une fleuriste dans les nouveaux Länder ou à peu près ce que gagne un ouvrier spécialisé dans la branche papier. Effet pervers probable : la possibilité de cumul ouverte par la loi Hartz IV ne devrait guère inciter au retour sur le marché du travail.

"Notre système actuel est inefficient et n'est plus finançable à terme », expliquait Wolfgang Clement (SPD), ministre de l'Economie et du Travail lors d'une conférence de presse (01-07-04). Le coût du régime que modifie la réforme était énorme : en 2003, le gouvernement fédéral a dépensé 16,7 milliards $€$ au titre de l'Arbeitslosenhilfe; les communes, quelque 9,5 milliards $€$ pour la Sozialhilfe. La première prestation était versée durant 26 mois en moyenne, la seconde, pendant 28 mois. Selon le ministère, l'ensemble des dépenses publiques allemandes consacrées aux mesures en faveur de l'emploi s'élevait en 2002 à plus de 71 milliards $€$ - un ordre de grandeur qui correspond à la moitié des dépenses de l'assurance maladie ou à un petit tiers du budget des retraites. Dans son projet de budget 2005, le gouvernement a programmé 26 milliards $€$ au seul titre de l'assistance aux chômeurs de longue durée.

II faut y ajouter 3,3 milliards $€$ destinés à couvrir les frais générés par la mise en place du nouveau dispositif dans lequel les demandeurs s'adresseront à un guichet unique (Job Center). Si le Bund (via l'Agence de Nuremberg et ses structures) assure les mesures de placement ou insertion, ainsi que le versement du minimum garanti, les communes restent, elles, compétentes pour l'aide au logement, les structures d'accueil à la petite enfance ou le suivi médical et psychologique des bénéficiaires dans la mesure où elles sont également en charge de l'aide sociale. Ce schéma implique la coopération entre services sociaux communaux et antennes locales de l'ANPE allemande ; elle sera testée dans une série de projets pilotes. Parallèlement, 72 communes ont choisi de gérer seules le nouvel Arbeitslosengeld II. Hartz IV aura aussi contribué au renforcement des compétences communales $-\mathrm{y}$ compris budgétaires.
Un salaire d'appoint et des Ein-Euro-Jobs

Une nouvelle

trappe à inactivité ?

71 milliards $€$ pour l'ensemble de la politique de l'emploi en 2002

Coopération entre communes et agences pour l'emploi 
Des critiques nourries par une mauvaise communication

La difficile acceptation des inégalités territoriales
Somme toute, le nouveau régime ne "démantèle » nullement l'Etat social allemand. Alors, pourquoi cristallise-t-il tant d'opposition? "C'est la preuve incontestable qu'il ne s'agit pas d'aménagements de façade », analyse Rüdiger Pohl, directeur de l'institut IWH de Halle spécialisé dans l'étude de l'économie des Länder est-allemands. "L'opposition aux réformes, très énergiquement propagée par une partie du monde syndical ou associatif, mais aussi des personnalités politiques de diverses obédiences, révèle combien sont nombreux dans la société ceux qui refusent de reconnaître que leurs acquis sont remis en question par la compétition globale des sites, et non pas par une politique de réformes abusivement qualifiée 'd'inéquitable' ». II est vrai que communication négligeante du gouvernement sur la loi Hartz IV avait prêté le flanc à une désinformation systématiquement entretenue par une singulière alliance entre extrêmes politiques, mouvements anti-mondialistes, syndicats clientélistes et membres des gouvernements est-allemands. Les élections régionales de septembre en Sarre, dans le Brandebourg et en Saxe, de même que les communales en Rhénanie du Nord-Westphalie n'ont guère incité non plus à la sérénité.

Les détracteurs se focalisent surtout sur la différence de $14 €$ entre le minimum garanti versé à l'est et à l'ouest - une inégalité de traitement injustifiée selon eux. II est vrai que, pour la première fois, une mesure politique rompt avec la pratique égalitaire qui prévalait jusqu'ici dans la politique de soutien aux nouveaux Länder. Mais la Commission Dohnanyi chargée du suivi de la transition est-allemande rappelle que, si les salaires est-allemands n'atteignent que près de $90 \%$ du niveau de l'ouest (pour une productivité de $68 \%$ seulement), le « niveau de vie matériel » n'est que de $80 \%$. Ce sont bel et bien les disparités régionales qui légitiment la différence de $14 €$. Habitués à être massivement soutenus par l'ouest (1250 milliards $€$ de transferts depuis l'unité, dont les trois quarts destinés au système de protection sociale), les Allemands de l'Est redoutent aussi la prochaine réduction des aides versées par l'ouest (l'Aufbau Ost prend fin en 2019) et par l'UE ; les fonds structurels (voir REA 61/03) iront alors majoritairement aux dix nouveaux Etats membres. Les disparités territoriales nord-sud, est-ouest, sont pourtant une des caractéristiques de l'Allemagne, rétorque le président fédéral Horst Köhler (magazine Focus, 13-09-2004). « Celui qui cherche à les égaliser consolide un Etat-subvention et lègue à la jeune génération une dette insupportable. II nous faut rompre avec cet Etat qui subventionne tout ».

LA REFORME ROMPT AVEC L'EGALITARISME qui avait fini par supplanter le principe d'équité au fondement de l'économie sociale de marché. Voilà pourquoi elle suscite une telle fronde : c'est ni plus ni moins une révolution culturelle. Primauté à l'effort individuel - telle est la devise de l'ensemble des réformes de l'Agenda 2010, toutes placées explicitement au service de l'innovation et de la compétitivité en fidélité à la stratégie adoptée en 2000 à Lisbonne par les Etats membres de l'UE. Retraites, santé, chômage - dans son discours inaugural (02-07-2004), le président Horst Köhler (CDU) apportait son plein soutien au gouvernement SPD/Verts : " l'Etat social dans sa configuration actuelle a présumé de ses moyens... II nous faut changer de mentalité, pour trouver un nouvel équilibre entre la responsabilité individuelle et la protection collective ».

\section{Indications bibliographiques}

BouRgeols I., " Le débat sur les trappes à inactivité en Allemagne », Regards sur l'économie allemande, $\mathrm{n}^{\circ} 51 / 01$

GRAF LAMBSDORFF O., Konzept für eine Politik zur Überwindung der Wachstumsschwäche und zur Bekämpfung der Arbeitslosigkeit, Bonn, 1982

LESTRADE B., « Bilan de la politique de l'emploi du gouvernement Schröder », Regards sur l'économie allemande, $n^{\circ}$ 59/02

PoHL R., «Agenda 2010 nach einem Jahr: die Reformen sind besser als ihr Ruf - ein Kommentar », IWH, Wirtschaft im Wandel, 5/2004

www.bundesregierung.de 